\title{
Observations and modelling of a large optical flare on AT Microscopii
}

\author{
D. García-Alvarez ${ }^{1}$, D. Jevremović ${ }^{1,2}$, J. G. Doyle ${ }^{1}$, and C. J. Butler ${ }^{1}$ \\ 1 Armagh Observatory, College Hill, Armagh BT61 9DG N. Ireland \\ e-mail: djc@star.arm.ac.uk, jgd@star.arm.ac.uk, cjb@star.arm.ac.uk \\ 2 Astronomical Observatory, Volgina 7, 11070 Belgrade, Yugoslavia
}

Received 26 September 2001 / Accepted 5 December 2001

\begin{abstract}
Spectroscopic observations covering the wavelength range 3600-4600 $\AA$ are presented for a large flare on the late type M dwarf AT Mic (dM4.5e). A procedure to estimate the physical parameters of the flaring plasma has been used which assumes a simplified slab model of the flare based on a comparison of observed and computed Balmer decrements. With this procedure we have determined the electron density, electron temperature, optical thickness and temperature of the underlying source for the impulsive and gradual phases of the flare. The magnitude and duration of the flare allows us to trace the physical parameters of the response of the lower atmosphere. In order to check our derived values we have compared them with other methods. In addition, we have also applied our procedure to a stellar and a solar flare for which parameters have been obtained using other techniques.
\end{abstract}

Key words. stars: activity - stars: chromospheres - stars: flare - stars: late-type

\section{Introduction}

Stellar flares are events where a large amount of energy is released in a short interval of time, radiating at almost all frequencies in the electromagnetic spectrum. Flares are believed to result from the release of magnetic energy stored in the corona through reconnection (see reviews by Mirzoyan 1984; Butler 1991; Haisch et al. 1991; Garcia Alvarez 2000). Many types of cool stars produce flares (Pettersen 1989), sometimes at levels several orders of magnitude more energetic than their solar counterparts. The exact mechanism(s) leading to the energy release and subsequent excitation of various emission features remains poorly understood. In the dMe stars (or UV Cet type stars) optical flares are a common phenomenon. In more luminous stars, flares are usually only detected through UV or X-ray observations (Doyle et al. 1989b), although optical flares have been detected in young early $\mathrm{K}$ dwarfs like LQ Hya (Montes et al. 1999).

One would like to trace all the energetic processes in a flare to some common origin likely to be localized at some unresolvably fine scale in the magnetic field. The largest solar flares observed involve energies of $\sim 10^{32}$ erg (Gershberg 1989), while large flares on dMe stars can be one order of magnitude larger

Send offprint requests to: D. García-Alvarez,

e-mail: dga@star.arm.ac.uk
(Doyle \& Mathioudakis 1990; Byrne \& McKay 1990). Even more energetic flares occur on the RS CVn binary systems, the total flare energy in the largest of this type of systems may exceed $E \sim 10^{38}$ erg (Doyle et al. 1992; Foing et al. 1994). Such a change in the star's radiation field modifies drastically the atmospheric properties over large areas, from photospheric to coronal layers. Models by Houdebine (1992) indicate that heating may propagate down to low photospheric levels, with densities higher than $10^{16} \mathrm{~cm}^{-3}$, although electrons with energies in the $\mathrm{MeV}$ range would be required to attain such depth.

As regards the derivation of physical parameters such as electron density and temperature, various methods have been used. For example, Katsova (1990) published an analysis of flare Balmer decrements. The broadening and merging of higher Balmer lines, dominated by the Stark effect, have also been used to estimate electron densities in the chromosphere (Donati-Falchi et al. 1985). Measurements of the broadening of the lower Balmer lines, which are less affected by the Stark effect, together with line shifts, provide information on the large scale motions during flares. Steep decrements are evidence for electron densities between $10^{8}$ and $10^{12} \mathrm{~cm}^{-3}$, while a shallow Balmer decrement indicates densities larger than $10^{13} \mathrm{~cm}^{-3}$ in the flaring $\mathrm{M}$ dwarf atmosphere.

Jevremović et al. (1998) and Jevremović (1999) developed a procedure to fit the Balmer decrement based on 
the solution of the radiative transfer equation using the escape probability computing technique of Drake (1980) and Drake \& Ulrich (1980) and the direct search method of Torczon $(1991,1992)$.

In this paper we present the results of a large flare observed on AT Mic (Gliese 799B, $V=10.25, B=11.83$ $20^{\mathrm{h}} 41^{\mathrm{m}} 51^{\mathrm{s}},-32^{\mathrm{o}} 26^{\prime} 07^{\prime \prime}$, Equinox 2000). It is a visual binary star with a separation of 4.0 arcsec Wilson (1978), approximately $10.2 \mathrm{pc}$ away, with both components of spectral type dM4.5e and subject to flaring (Joy \& Wilson 1949). Kunkel (1970) determined the flare incidence at 2.8 flares per hour. From its position in the $(M v, R-I)$ diagram, Kunkel (1973) deduced that AT Mic lies slightly above the main sequence, pointing to its probable membership of the young disc population. Nelson et al. (1986) reported $29 U$-band/ $B$-band flares in 39.3 hours, only one of which showed microwave flaring at $5 \mathrm{GHz}$. They also reported two microwave bursts without any optical counterpart. Kundu et al. (1987) found that both components of the AT Mic system were active and variable at 6 and $20 \mathrm{~cm}$ wavelengths. The peak emission levels were detected in the southern component (Gliese 799B). X-ray and ultraviolet emission from this system has been observed several times over the last ten years or so (Linsky et al. 1982; Pallavicini et al. 1990).

In Sect. 2 we present the current dataset plus a discussion of the data analysis while Sect. 3 gives the results. In Sect. 4, we analyse the evolution of the main physical parameters during the flare using the code developed by Jevremović et al. (1998) and apply the procedure to a stellar and a solar flare for which parameters have been obtained using other techniques. The conclusions are given in Sect. 5.

\section{Observations and data reduction}

The observations were carried out at the South African Astronomical Observatory between 20-26 August 1985. Optical spectroscopy was obtained with the $1.9 \mathrm{~m}$ telescope at SAAO equipped with the RGO Unit Spectrograph and the Reticon Photon Counting System (RPCS), providing spectral coverage from 3600 to $4600 \AA$ at a reciprocal dispersion of $50 \AA / \mathrm{mm}$ and a wavelength resolution of $\Delta \lambda \sim 2 \AA$ (FWHM) at $\mathrm{H} \gamma$. The spectral region includes the entire Balmer series except $\mathrm{H} \alpha$ and $\mathrm{H} \beta$, as well as the CaII H \& K lines and the He I $4026 \AA$ and He I $4471 \AA$ lines. The exposure time was $60 \mathrm{~s}$ which with readout overheads gave a time resolution of $70 \mathrm{~s}$. During the run on AT Mic, sky conditions were generally good although seeing was variable.

Spectra were reduced in the standard manner, consisting of background subtraction and flat-field correction using exposures of a tungsten lamp. Wavelength calibration was made with $\mathrm{Cu}-\mathrm{Ar}$ lamp spectra taken immediately preceeding and following the stellar spectra. A first-order spline cubic fit to some 16 lines achieved a nominal wavelength calibration accuracy of $0.12 \AA$. An atmospheric extinction correction for SAAO was also applied to the data.

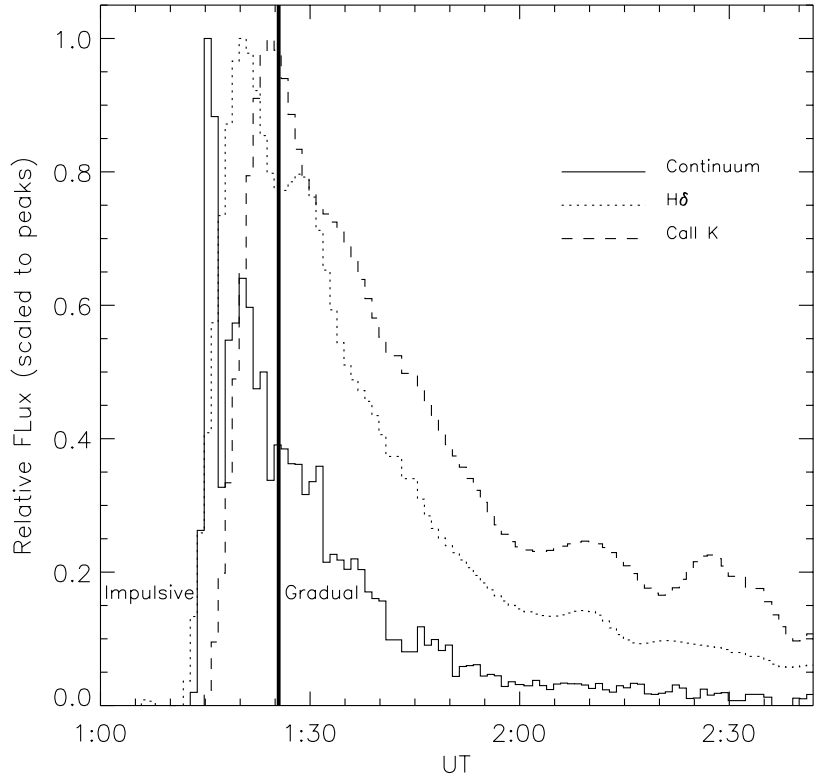

Fig. 1. Normalised flare-only light curves for H $\delta$, Ca II K and the continuum (3600-3700 $\AA$ ). The demarcation between the impulsive and gradual phases, taking into account the different line behaviour, is indicated by a vertical line.

Flux calibration, by reference to spectrophotometric standard stars (LTT7987 and LTT9239), was carried out using the NOAO packages IRAF ${ }^{1}$. The observations were made in spectrophotometric mode with a slit of $400 \mu$ width ( $\sim 4$ arcsec), adequate to encompass the entire image during the worst seeing conditions encountered during the run. A dekker was selected which provided an approximately square aperture. The programme star (AT Mic) and the spectrophotometric standards were observed under similar conditions. From a comparison of the calibrations afforded by the two standards, we estimate the spectrophotometric accuracy to vary from $\pm 0.12 \mathrm{mag}(\sim 12 \%)$ at $3600 \AA$ to $\pm 0.03 \mathrm{mag}(\sim 3 \%)$ at $4600 \AA$. The fluxes of individual emission lines will have additional errors associated with the photon noise level of the binned fluxes.

\section{AT Mic flare at 1:20 UT on 21 August 1985}

In this paper we report on an energetic flare, lasting more than one and a half hours, observed on 21 August 1985. The continuum emission, which initially peaked in the impulsive phase at 1:15 UT, had a second peak at 1:20 UT, roughly coincident with the primary peak in the Balmer lines. In addition, there is evidence for a second peak at 1:30 UT in some of the Balmer lines $(\mathrm{H} \delta, \mathrm{H} 8)$ and also in He I $4026 \AA$. Figure 1 shows a rapid continuum flux rise and decay, while the hydrogen Balmer lines peak later and decay more slowly. There is some indication that the low-order Balmer lines decay more slowly than the

\footnotetext{
${ }^{1}$ IRAF is distributed by the National Optical Astronomy Observatories, which are operated by the Association of Universities for Research in Astronomy, Inc., under cooperative agreement with the National Science Foundation.
} 
high-order lines, as has been noted by Butler (1991) in a flare on YZ CMi. The CaII K line rises and decays even more slower than the hydrogen lines. Following Hawley \& Pettersen (1991), we define the start of the gradual phase as the time when the continuum shows a turnover from fast to slow decay. The change from impulsive to gradual phase is indicated in Fig. 1 with a solid vertical line.

Many emission lines other than the Balmer line series and $\mathrm{Ca} I \mathrm{I} \mathrm{H} \& \mathrm{~K}$ were seen during this flare, e.g. Fe I and HeI. Many of these were visible only during the impulsive phase and at the beginning of the gradual phase. Observed at higher spectral resolution these lines would provide important information on the changing atmospheric structure and hence flare heating. Figure 2 illustrates a time sequence of the optical flare-only spectra obtained during the AT Mic flare.

In order to form a mean quiescent spectrum we have co-added 75 quiescent spectra. The Balmer lines from $\mathrm{H} \gamma$ to $\mathrm{H} 13$ and the Ca II H \& K lines are visible and in emission in the quiescent spectrum. In the flare spectrum the Balmer lines increase their emission and there is a substantial enhancement of the ultraviolet continuum. There is also some evidence for a filling in of the strong absorption line CaI at $4227 \AA$ observed during the impulsive and gradual phase of the flare. The He I $4026 \AA$ and He I $4471 \AA$ lines are also in emission during the flare.

Emission line fluxes were measured as a function of time for the important contributors to the radiative losses in the optical, including the hydrogen Balmer lines from $\mathrm{H} \gamma$ to $\mathrm{H} 9$, the Ca II $\mathrm{H} \& \mathrm{~K}$ lines and the helium lines (see Table 1). To analyze the optical spectra, the mean quiescent spectrum was first subtracted from the spectra taken during the flare. The emission line fluxes were then determined by numerically integrating the flux under the line, using carefully chosen windows to set the local continuum level. Our low resolution spectroscopy cannot resolve the Ca II $\mathrm{H}$ and $\mathrm{H} \epsilon$ line, but the blend is dominated by the behavior of $\mathrm{H} \epsilon$. We note the comparatively slow response of the Ca II $\mathrm{K}$ line compared to the Balmer lines.

Both the Balmer lines and CaII emission lines were above the quiescent values for more than 2 hours, while the continuum enhancement lasted around one hour.

\subsection{Line broadening}

Near flare maximum, the wings of $\mathrm{H} \alpha, \mathrm{H} \delta, \mathrm{H} 8$ and even H9 are very broad, around $25 \AA$ at the base, as is shown in Fig. 3. As a result, the Balmer lines start to merge at about H13; meanwhile CaII K shows very little broadening. Giampapa (1983) and Worden (1983) pointed out that during flares the lower Balmer lines such as $\mathrm{H} \alpha$ and $\mathrm{H} \beta$ were unbroadened, while the higher Balmer series lines show significant broadening, $20 \AA$ or more for some lines. They also reported that the Balmer lines (and HeI) in a very large flare were broadened and asymmetric to the red, while CaII is unbroadened. Balmer line broadening in solar flares is generally attributed to Stark broadening (Švestka 1972).
In order to obtain an estimate of the broadening of the hydrogen lines during the flare, we have measured the full width at half maximum (FWHM) for $\mathrm{H} \gamma, \mathrm{H} \delta, \mathrm{H} 8$ and CaII K. The FWHM for the Balmer lines were obtained using a Lorentzian profile, while a Gaussian profile was used for CaII K. From Fig. 4 we can conclude that the FWHM of the emission lines increases dramatically during the flare for the Balmer lines, although for CaII K there is almost no change.

It seems that line broadening continues well into the gradual phase of the flare, finishing around the same time as the continuum enhancement. As Doyle et al. (1988) pointed out, a possible explanation for the line wing enhancement could be motions of several hundred $\mathrm{km} \mathrm{s}^{-1}$. If that were the case in the AT Mic flare, it would require the amount of radiating material moving upwards and downwards to be approximately equal throughout the flare in order to produce the observed profiles. Although this could be the case it has not been observed on the Sun, where normally a pronounced redshift in $\mathrm{H} \alpha$ is usually observed during the impulsive phase (Zarro \& Canfield 1989; Wuelser \& Marti 1989).

If hydrodynamic effects were contributing significantly in the chromospheric line forming regions, we would expect Ca II $\mathrm{K}$ to show similar broadening to the Balmer lines, but this was not observed. Although the broadening of the Balmer series lines decreases almost exponentially during and after the impulsive phase, the central line intensity of $\mathrm{H} \delta$ (Fig. 1) shows two peaks, indicating that there are two energy releases. The first burst induced line broadening while the second burst did not, suggesting that this flare may have derived from an arcade of loops.

The excess broadening of the Balmer lines could be interpreted as turbulence as we did not observe any clear asymmetry at any time, in any line, during the flare.

Our conclusions in the last two sections, regarding the evolution and broadening of the emission lines during the flare on AT Mic, are similar to those of Hawley \& Pettersen (1991) for a flare on AD Leo.

\subsection{The HeI $4026 \AA$ and Hel $4471 \AA$ triplet lines}

Helium lines are produced under generally higher excitation conditions than other lines in the visible and the lines are generally optically thin, which makes interpretation easier. The helium lines are also useful as indicators of the presence of electric fields in the flaring plasma due to their forbidden components. However, even for the Sun, exact knowledge of the background spectrum is required for the determination of the contribution of the forbidden lines. The He I lines have been reported in solar white light flares (Lites et al. 1986) and in stellar flares (Moffett \& Bopp 1976; Doyle et al. 1988). Lites et al. (1986) pointed out that any mechanism which produces a dense plasma at relatively high temperatures $\left(2 \times 10^{4}-10^{5} \mathrm{~K}\right)$ will produce emission in neutral helium.

The He I $4026 \AA$ line is a diffuse triplet between levels $2^{3} \mathrm{P}$ and $5^{3} \mathrm{D}$. This line is usually not detected except in 

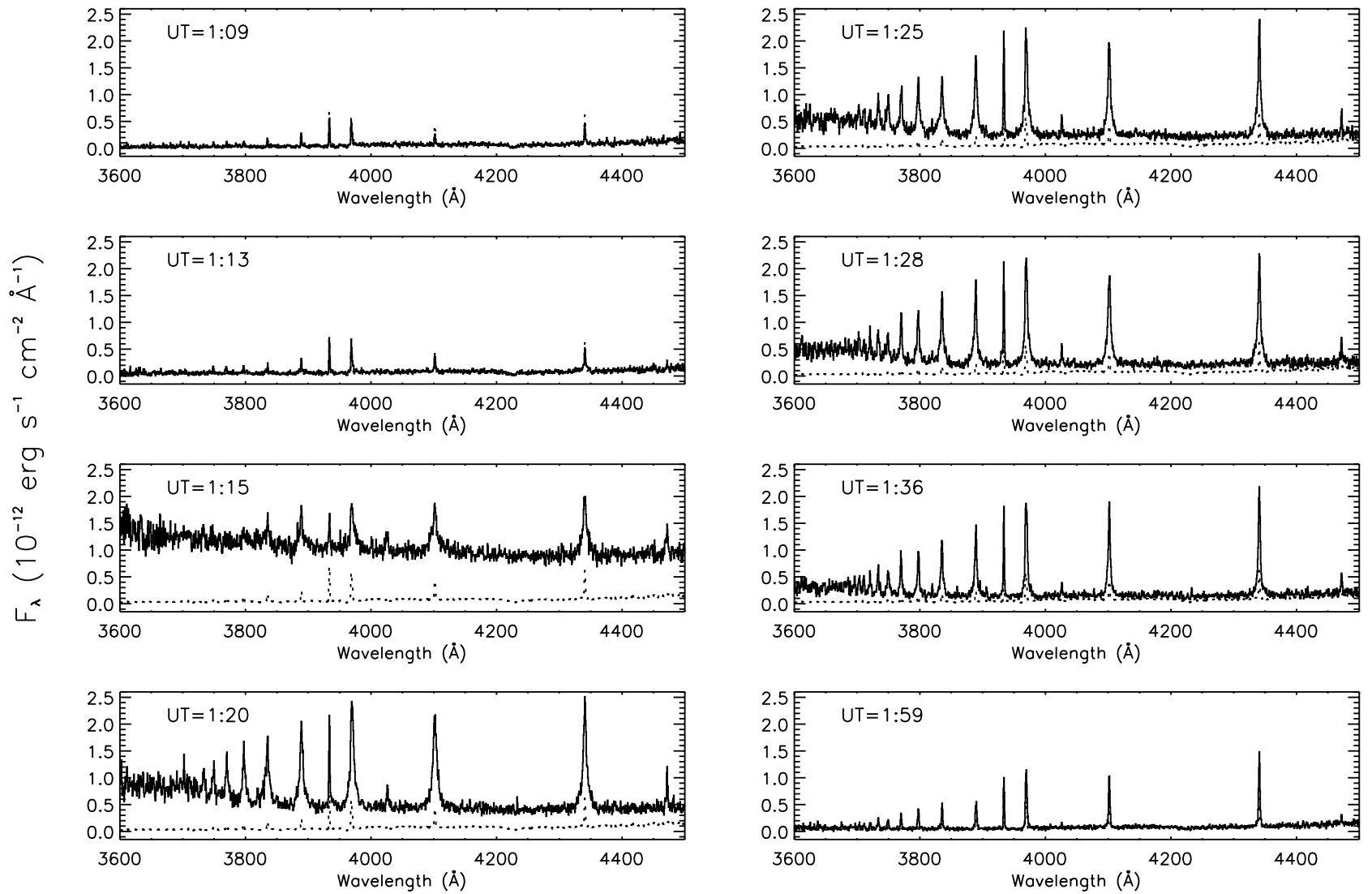

Fig. 2. The time sequence of optical flare-only spectra of AT Mic during the pre-flare phase, impulsive phase, maximum continuum, maximum flux in $\mathrm{H} \delta$, minimum between the two maxima, second maximum flux in $\mathrm{H} \delta$, the beginning of the gradual phase and finally the gradual phase. The mean quiescent spectrum is shown as a dotted line for comparison.

Table 1. The total energy radiated in the Balmer, calcium and helium lines during the flare on AT Mic. Units are $10^{30}$ erg.

\begin{tabular}{|c|c|c|c|c|c|c|c|c|c|c|}
\hline$\overline{\mathrm{UTT}}$ & $\overline{\mathrm{H} \gamma}$ & $\overline{\mathrm{H} \delta}$ & $\overline{\mathrm{H} 8}$ & H9 & H10 & 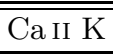 & $\overline{\mathrm{C}} \mathrm{Ca}$ II $\mathrm{H}+\mathrm{H} \epsilon$ & $\overline{\mathrm{CaI}}$ & He I $4026 \AA$ & 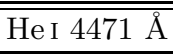 \\
\hline $1: 13$ & 1.61 & 1.42 & .74 & .46 & .24 & .04 & .36 & .04 & .52 & .90 \\
\hline $1: 15$ & 4.62 & 4.37 & 2.50 & 2.10 & .87 & .25 & 3.76 & .01 & 1.34 & 1.43 \\
\hline $1: 20$ & 11.07 & 10.69 & 7.54 & 5.28 & 2.83 & 2.28 & 9.86 & .36 & .67 & 1.24 \\
\hline $1: 25$ & 9.03 & 8.25 & 6.12 & 5.24 & 3.23 & 2.42 & 8.28 & .14 & .56 & .94 \\
\hline $1: 28$ & 8.12 & 8.52 & 6.32 & 4.91 & 2.84 & 2.60 & 8.10 & .41 & .61 & .90 \\
\hline $1: 30$ & 6.30 & 5.22 & 4.05 & 3.42 & 2.08 & 1.63 & 5.94 & .34 & .48 & .48 \\
\hline $2: 00$ & 1.81 & 1.55 & .97 & .85 & .65 & .51 & 1.85 & .11 & .13 & .39 \\
\hline
\end{tabular}

the strongest events. It was shown (e.g. Vidal 1964) that the higher members of this triplet are quite sensitive to density changes. The He I $4026 \AA$ line is near to a strong Mn I absorption feature at $4030 \AA$ (which during intense activity can go into emission due to pumping from $\mathrm{Mg}$ II k, (Doyle et al. 1992, 2001)) thus making detection difficult. We observed the He I $4026 \AA$ line and He I $4471 \AA$ line in emission during the impulsive phase and well into the gradual phase of the flare on AT Mic (Fig. 5). Two peaks are seen in the time profile of He I $4026 \AA$, the first at 1:20 UT and the second at 1:30 UT. These are simultaneous with the maxima in the Balmer lines. In He I $4471 \AA$ only a single peak at 1:20 UT is visible.
The FWHM of the two He I lines are $5.0 \AA$ and $2.8 \AA$ respectively. Gieske \& Griem (1969) give a procedure for computing the line profile of He I $4026 \AA$ including Stark and Doppler broadening. With their model for $T_{\mathrm{e}}=20000 \mathrm{~K}$, we derive an upper limit for the electron density of $10^{16} \mathrm{~cm}^{-3}$ for the He I emitting region.

\subsection{Continuum analysis}

We have measured the fluxes (Table 2) for three different continuum regions: one in the near ultraviolet, 3600$3700 \AA$ and two in the blue, 4130-4300 $\AA$ (with the exclusion of the Ca I line region) and 4435-4545 A. Figure 6 

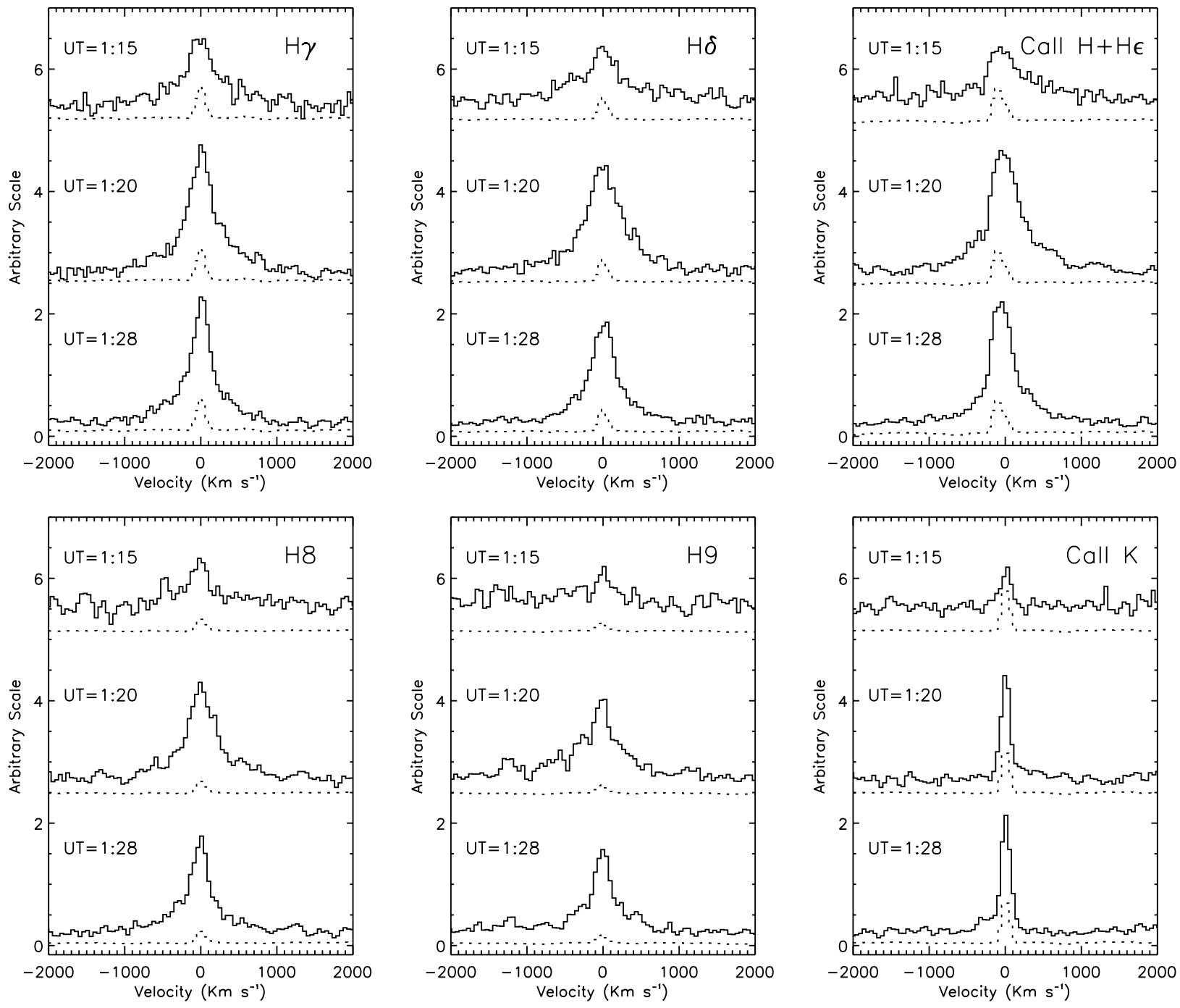

Fig. 3. Comparison of the mean quiescent and flare-only line profile for the Balmer series lines, Ca II $\mathrm{H}+\mathrm{H} \epsilon$ and Ca II K.

shows the variation in the ratios of the continuum fluxes during the impulsive and gradual phases of the flare. We note that the ratios of the near ultraviolet band to the two longer wavelength bands do not reach their maximum blueness until the end of the impulsive phase and the beggining ot the gradual phase (indicated by the vertical line), whereas the ratio of the intermediate wavelength band to the longer wavelength band reaches it maximum blueness at the beginning of the impulsive phase. As all three continuum bands reach their maximum brightness at the start of the impulsive phase, this behaviour suggest that a simple heating of a static, confined, kernel with radiation following a black-body law, is not appropiate (see Butler 1996).

Bopp \& Moffett (1973) suggested that the continuum enhancement ends when the fast decay (what we term the impulsive phase) has ended. In fact, this generally accepted notion, that the white-light continuum is present only during the impulsive phase is certainly not the case for the AT Mic flare nor for the flare on AD Leo reported by Hawley \& Pettersen (1991).
Spectroscopic observations of $\mathrm{M}$ dwarf flares reveal the shape of the flare continuum and allow us to explore the possible continuous emission mechanisms. It has been suggested (e.g., Cram \& Woods 1982; Houdebine 1992) that the optical flare continuum is composed of bound-free and free-free emission from a two-component thermal model characterized by electron temperatures of $T \sim 10^{5} \mathrm{~K}$ and $T \sim 2 \times 10^{4} \mathrm{~K}$. Cram \& Woods (1982) showed the spectral energy distributions of some of the suggested radiative processes that may give rise to the flare continuum. The shape of the free-free curve at $T \sim 10^{5} \mathrm{~K}$ obtained by Giampapa (1983) produces reasonable fits to the observed flare continuum. Kunkel (1970) has argued that hydrogen free-bound emission is the principal component for the observed continuum emission in $M$ dwarf flare events. The curve for this process given by Giampapa (1983) only fits the red part of the continuum, which would imply that additional continuum processes, other than bound-free, are taking place in higher temperature layers. Hawley \& Fisher (1992) fitted the observed flare continuum, but the results were much bluer than those computed from the 

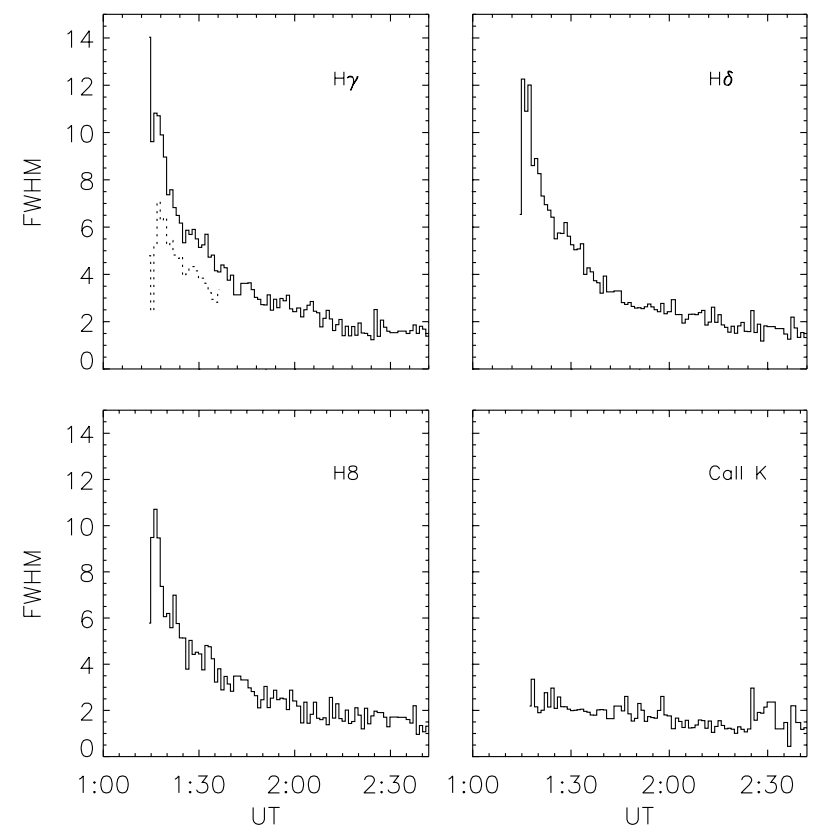

Fig. 4. FWHM through the flare-only spectrum on AT Mic for $\mathrm{H} \gamma, \mathrm{H} \delta, \mathrm{H} 8$ and $\mathrm{Ca}$ II $\mathrm{K}$ lines. For the Balmer lines we have used a Lorentzian profile and Gaussian profile for the Ca II K line. In the top left we plot the Voigt profile fitting (dotted) for $\mathrm{H} \gamma$.
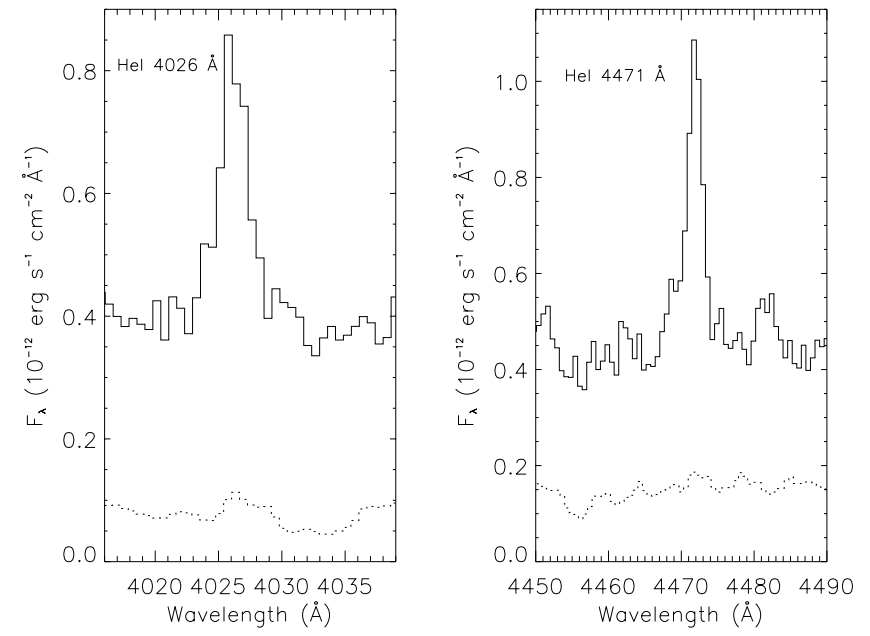

Fig. 5. Left panel: average over 4 flare-only spectra of He I $4026 \AA$ around flare maximum. Right panel: He I $4471 \AA$ average over 4 flare-only spectra around flare maximum.

models. They concluded that the best fit to their observations was a blackbody spectrum with $T \sim 8500-9500 \mathrm{~K}$. Hawley \& Fisher (1992) proposed that the flare continuum is formed by photospheric reprocessing of intense UV/EUV line emission from the upper chromosphere.

Dame \& Vial (1985) proposed a model for solar whitelight flares in order to explain the observed blue continuum. Using the study of radiative processes in solar flares (Dame \& Cram 1983) they show how existing semiempirical models can be modified to predict a visible spectrum more similar to the WLFs emission observed. The hot temperature plateau $\left(6-9 \times 10^{4} \mathrm{~K}\right)$ model proposed

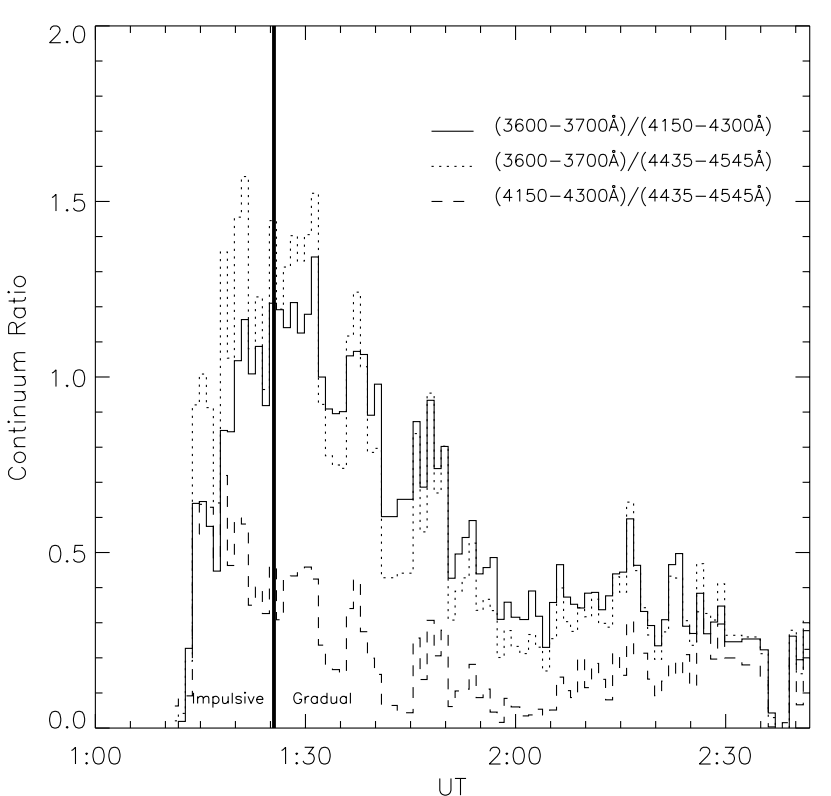

Fig. 6. Continuum flux ratios through the flare on AT Mic. It is clear from the plot that the continuum of the flare has a sustantial blue component during both the impulsive phase and gradual phase and that the maximum blueness in the near UV occurs close to the end of the impulsive phase.

Table 2. Continuum energy radiated during quiescence and during flare on AT Mic. Units are $10^{32} \mathrm{erg}$.

\begin{tabular}{cccc}
\hline \hline UT & $3600-3700 \AA$ & $4150-4300 \AA$ & $4435-4545 \AA$ \\
\hline Quiescence & .03 & .05 & .12 \\
$1: 13$ & .04 & .05 & .11 \\
$1: 15$ & .98 & .68 & .74 \\
$1: 20$ & .63 & .31 & .35 \\
$1: 25$ & .40 & .17 & .22 \\
$1: 28$ & .37 & .16 & .21 \\
$1: 36$ & .23 & .11 & .16 \\
$2: 00$ & .06 & .06 & .12 \\
\hline
\end{tabular}

by Dame \& Vial (1985) is not valid for all WLFs but provides an explanation for the origin of an unidentified source of opacity distinct from the Balmer and Paschen continuum. Houdebine et al. (1996) suggested that this blackbody type emission is due to the enhanced electron density in the lower chrosmophere.

However, the blue continuum enhancement could also be due to merging of the higher members of the Balmer lines particularly in the vicinity of the Balmer jump, which indicates that these lines contribute a non-negligible amount to the near-ultraviolet continuum (Donati-Falchi et al. 1985; Zarro \& Zirin 1985). One case has been reported in which the continuum was found to be red in color during the first stages of the flare (Doyle et al. 1989a).

\section{Derivation of flare parameters}

We normalized the flare-only fluxes for $\mathrm{H} \delta, \mathrm{H} 8, \mathrm{H} 9$ and $\mathrm{H} 10$ to $\mathrm{H} \gamma$ at several times during the impulsive and 
Table 3. Comparison of physical parameters for flares on YZ CMi and on the Sun obtained with the different codes.

\begin{tabular}{lcccccc}
\hline \hline Star & Code applied & Reference & $N_{\mathrm{e}}\left(\mathrm{cm}^{-3}\right)$ & $T_{\mathrm{e}}(\mathrm{K})$ & $\tau(\mathrm{Ly} \alpha)$ & Area $\left(\mathrm{cm}^{2}\right)$ \\
\hline YZ CMi & Our Code & 1 & $5 \times 10^{13}$ & 13000 & $3 \times 10^{6}$ & $1 \times 10^{18}$ \\
& Gas Dynamic & 2 & $10^{14}$ & 10000 & $2 \times 10^{6}$ & $5 \times 10^{17}$ \\
\multirow{3}{*}{ The Sun } & Our Code & 1 & $2 \times 10^{14}$ & 18000 & $7 \times 10^{4}$ & $7 \times 10^{19}$ \\
& Stark Broadening & 3 & $(2-3) \times 10^{13}$ & 14000 & $\mathrm{n} / \mathrm{a}$ & $5 \times 10^{19}$ \\
\hline
\end{tabular}

References: 1: This work, 2: Katsova et al. (1991), 3: Johns-Krull et al. (1997).

gradual phases. We do not include H11 and higher Balmer lines due to the difficulty in assigning the local continuum level. The Balmer decrement became quite shallow and stayed nearly constant during the impulsive phase of the flare and well into the gradual phase. After this, the decrement steepens but does not become as steep as that during the quiescent phase.

\subsection{Our procedure}

Jevremović et al. (1998) developed a procedure to fit the Balmer decrements. It is based on the solution of the radiative transfer equation, using the escape probability technique of Drake (1980), and Drake \& Ulrich (1980). The Drake \& Ulrich (1980) method assumes: (i) that the escape probability for a slab of optical thickness is averaged over all directions with a uniform distribution of emitters, (ii) complete redistribution of frequencies and (iii) that the line profile is represented by a Doppler core and power law wings of slope $-5 / 2$ to emulate the Stark effect. Jevremović et al. (1998) adopted a simplified picture of the flaring plasma as a slab of hydrogen with an underlying thermal source of radiation and which causes photoionization. This source, which can be considered to be the photosphere, can be exposed to additional heating during the flare. It assumes that the Balmer lines are formed in the upper chromosphere. We note that, this simplified picture leaves out some important processes (i.e. cooling from $\mathrm{Mg}$ II, CaII and other metals). However, as seen from Table 1 the AT Mic flare is predominantly seen in Balmer lines and we believe that this picture may be applicable. The procedure minimizes the difference between the observed and calculated Balmer decrement using a multi-directional search algorithm (Torczon 1991, 1992). This allows us to find the best possible solution for the Balmer decrement in four parameter space where the parameters are: electron temperature, electron density, optical depth in the Ly $\alpha$ line and the temperature of the underlying source. Details of the procedure and the multisearch algorithm and comparison with the classical simplex algorithm are given in Jevremović et al. (1998). From the best solutions for the Balmer decrement, the procedure allows us to calculate the effective thickness of the slabs of hydrogen plasma with which we are able to determine the surface area of the emitting plasma from the calculated emission measure. Although the temperature of the underlying source could be a free parameter in our code, we have fixed a lower limit at $5500 \mathrm{~K}$ for the Sun and $2500 \mathrm{~K}$ for YZ CMi and AT Mic, for numerical stability reasons.

\subsection{Comparison with other codes}

In order to check the Jevremović et al. (1998) procedure we have applied it to two flares for which physical parameters have been independently derived, one stellar and one solar.

\subsubsection{Comparison with the gas-dynamic model for the YZ CMi flare}

We have applied our procedure to a flare on YZ CMi observed during a coordinated run involving telescopes at the South African Astronomical Observatory, and the ESA X-ray satellite, EXOSAT, on 4 March 1985. The photometric observations consisted of $U B V R I$ photometry with a $0.75-\mathrm{m}$ telescope. Details have been published by Doyle et al. (1988). The spectra of the flare on YZ CMi, over the wavelength range 3600-4400 $\AA$ were analysed by Katsova et al. (1991) using a gas-dynamic model. Based on the gas-dynamic model and using the data set for the flare observed by Doyle et al. (1988) on YZ CMi, Katsova et al. (1991) derived the main physical parameters at flare maximum. They matched quite well the observed data with the theoretical decrement for a temperature $T=10^{4} \mathrm{~K}$, electron density $10^{14} \mathrm{~cm}^{-3}$ and optical depth of the layer at the Ly $\alpha$ line center $\tau_{\mathrm{Ly} \alpha}=2 \times 10^{6}$. They also derived the emitting source area of $>5 \times 10^{17} \mathrm{~cm}^{2}$ using a description of the optical continuum as a Planck function for a temperature of $10^{4} \mathrm{~K}$.

Our values for the derived flare parameters are reasonably close to those values obtained by Katsova et al. (1991) (see Table 3), although our values for the electron temperature and Ly $\alpha$ optical depth at line center are slightly larger and smaller respectively. We obtained an effective thickness of the slab of hydrogen plasma of around $2000 \mathrm{~km}$ and an emissivity of $3.25 \mathrm{erg} \mathrm{cm}^{-3} \mathrm{~s}^{-1}$.

\subsubsection{Comparison with a Stark broadening model for a solar flare}

As a second check we have also applied our procedure to a solar flare observed on 6 March 1993. Details of the flare have been published by Johns-Krull et al. (1997). They fitted the observations with models that include Stark 


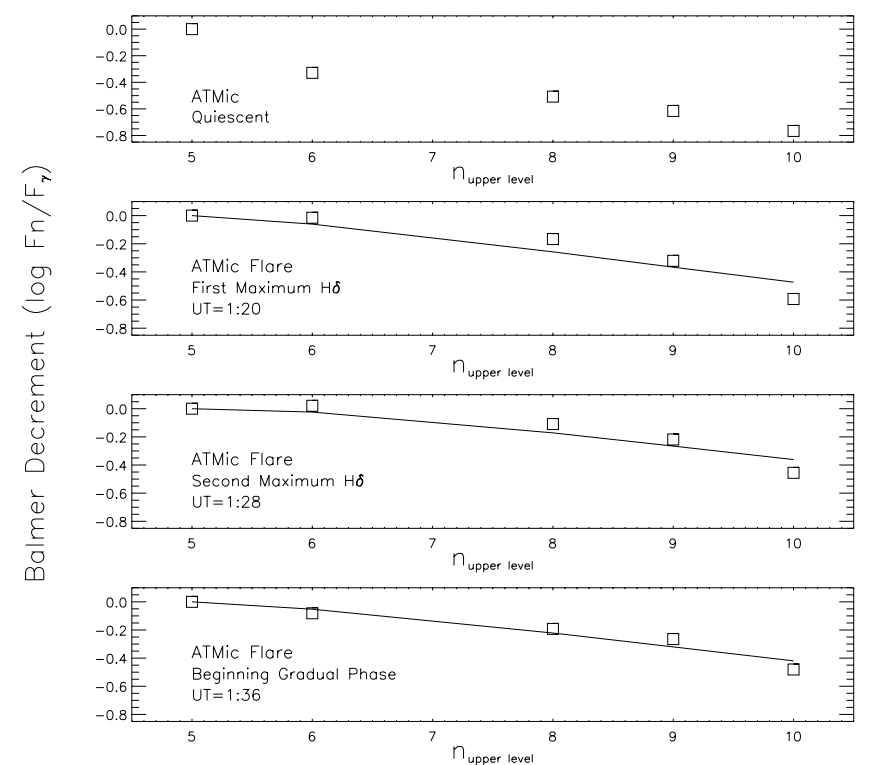

Fig. 7. The observed Balmer decrements (squares) and the optimum computed fits (solid line) during quiescence and at different times during the flare.

broadening by electrons, assuming that the Balmer lines form in a homogeneous flaring region overlying the quiet photosphere that has $n_{\mathrm{e}} \approx(2-5) \times 10^{13} \mathrm{~cm}^{-3}$. They modelled the line widths following the general procedure outlined by Redman \& Suemoto (1954) and Švestka (1963), which attempts to model the flare emission profile by taking into account the effects of self-absorption, Doppler broadening and Stark broadening, and comparing the resulting profiles with the observed flare-quiet Sun profiles. Johns-Krull et al. (1997) found that, over the course of the first 10 flare spectra, the electron density varied fairly randomly in the range $(2-3) \times 10^{13} \mathrm{~cm}^{-3}$. Meanwhile, the values for the electron temperature were around $14000 \mathrm{~K}$. Although they did not calculate the flare area they provided an $\mathrm{H} \alpha$ image taken at $\mathrm{BBSO}$ around $10 \mathrm{~min}$ after the flare maximum and from this image one can infer a solar flare area of about $5 \times 10^{19} \mathrm{~cm}^{2}$.

Using our procedure, and the solar flare data obtained by Johns-Krull et al. (1997), we obtain the parameters at solar flare maximum as shown in Table 3. As in the case of the YZ CMi flare, our values are close to those obtained by Johns-Krull et al. (1997) although our value for the electron density is almost one order of magnitude larger. This result for the electron density could be due to the fact that, in our fit of the solar flare, we have not fitted the lower Balmer lines, $\mathrm{H} \alpha$ and $\mathrm{H} \beta$ which are formed higher in the atmosphere, and were observed by Johns-Krull et al. (1997). We have also discarded H7 in our fitting, in order to compare the results of both solar and stellar flares based on the same Balmer lines. The flare area is in very good agreement with that derived from the observations. We obtained an effective thickness for the hydrogen slab of almost $100 \mathrm{~km}$ and an emissivity of $145 \mathrm{erg} \mathrm{cm}^{-3} \mathrm{~s}^{-1}$.

\subsection{Modelling the AT Mic flare}

From the above two cases we concluded that the procedure of Jevremović et al. (1998) gives reliable results and we proceed to apply it to the AT Mic flare. In Fig. 7 we have plotted the data and three Balmer decrement fits during the flare and one during quiescence. The evolution of the main parameters is given in Fig. 8.

Analysing the Balmer decrement during the AT Mic flare, we observed that, at the beginning of the impulsive phase, the Balmer decrement has a steep slope which become shallower as the flare progresses through its impulsive phase. The Balmer decrement keeps almost the same slope until well into the gradual phase, after which the slope steepens reaching similar values to that in the quiescent phase. In the two spectra prior to the first $\mathrm{H} \delta$ maximum at 1:20 and in the three spectra after the second $\mathrm{H} \delta$ maximum at $1: 28$, the $\mathrm{H} \delta$ flux is larger than the $\mathrm{H} \gamma$ flux. Due to their similarity, we have compared the observed Balmer decrement behaviour for the flare on AD Leo reported by Hawley \& Pettersen (1991) and the observed Balmer decrement for our flare on AT Mic. During the quiescent phase, the slope for AT Mic is steeper than for AD Leo, while, during the impulsive and gradual phases, the slopes for AT Mic are shallower than thoses for AD Leo. This means that the variations of the physical parameters during the AT Mic flare are larger than during the AD Leo flare.

From Fig. 8 one can see that there are three distinct peaks in the electron temperature, of which two coincide with peaks in $\mathrm{H} \delta$. We interpret these two peaks as the result of two sudden separate releases of energy (which presumably correspond to the two continuum peaks shown in Fig. 1), while the third peak in the temperature is connected to additional, more gradual heating. This is also seen in the behaviour of the temperature of the background source and in Fig. 6. Note that peaks in the slab thickness coincide with the maximum temperature, which can be interpreted by a change in the degree of ionization with temperature. An increase in the degree of ionization leads to a thicker slab for the same optical depth. Also, the thickness of the slab, coupled with the low emissivity of the plasma, explains the relatively low increase in the continuum/line flux during the third peak (see Fig. 1). For example, the modeled emissivity in $\mathrm{H} \gamma$ for the first peak was around $150 \mathrm{erg} \mathrm{cm}^{-3} \mathrm{~s}^{-3}$ while during the third event the emissivity was around $10 \mathrm{erg}^{-3} \mathrm{~cm}^{-3}$. Similar volumes of plasma for both peaks lead to much lower increase in the flux during the third event (contrary to what one would expect taking into account only thermal radiation). Also, one has to keep in mind that our background source is included only as the source of photoionization which controls ionization balance. Inclusion of other sources of ionization (i.e. focused beam of electrons/protons) would probably change the results of modeling, but that is beyond the scope of this paper.

We found little change in the electron density during the flare $(\mathrm{s})$ and it stays between $10^{13}-10^{14} \mathrm{~cm}^{-3}$. These 

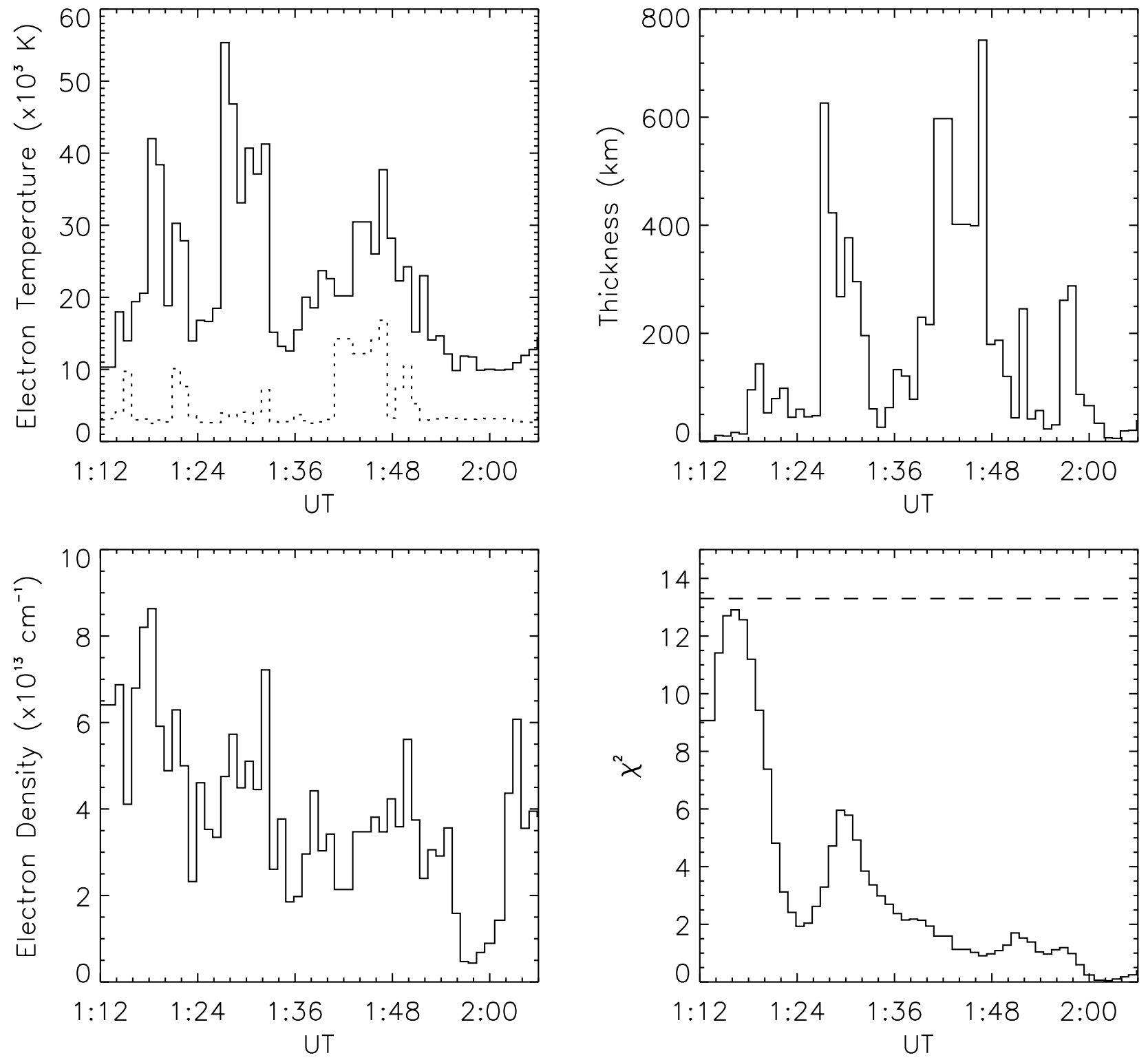

Fig. 8. Parameters derived from the fit to the Balmer decrement using the direct search method. Evolution of the electron temperature and temperature of background source are shown in the top left panel, electron density in bottom left panel, effective plasma thickness in top right panel and goodness of fit in bottom right panel.

values are one or two orders of magnitude below the upper limits deduced from either Stark broadening or the Inglis-Teller relation. This confirms the reliability in our method, based in the simultaneous analysis of a few lines, rather than some earlier estimates of electron density (i.e. Houdebine 1992), based on a single line analysis.

From the best fit solutions for the Balmer decrement, we have calculate the effective thickness of the slab of hydrogen plasma from the beginning of the impulsive phase until the beginning of the gradual phase as shown in the top right panel of Fig. 8. Using these results we are able to determine the surface area of the emitting plasma from the calculated emission measure and the measured fluxes in $\mathrm{H} \gamma$. From these results we conclude that the area of the flare reach three maximum values, which take place after each maxima in the electron temperature. The values we obtain are around few times $10^{19} \mathrm{~cm}^{2}$ for all of them. Taking into account that the radius of AT Mic is $0.32 R_{\odot}$, this suggests that the flares occupy around 0.6 percent of the stellar surface area. This is comparable with the sizes of the largest solar flares (Tandberg-Hanssen \& Emslie 1988).

\section{Conclusions and discussion}

We observed a major flare on AT Mic in the 3600-4500 wavelength region which allowed us to compute a detailed flare energy budget in the optical spectral region. The physical parameters obtained are consistent with previously derived values for stellar flares. We have compared the results from our method for two flares previously analysed, namely, one on YZ CMi and one on the Sun, obtaining reasonable agreement. 
We have obtained the first detailed trace of physical parameters during a stellar flare. We have also shown that our method could provide a suitable model for the study of flares on stars of different spectral type (G-M). The total radiated energy released in the region $3600-4500 \AA$ during the flare was $\sim 4 \times 10^{33} \mathrm{erg}$ and $\Delta U \sim 4 \mathrm{mag}$. The energy radiated by this flare is comparable with the strongest solar ones but is of medium strength compared to flares on other dMe stars.

Further stellar observations with high time and high spectral resolution should be obtained. Also, further solar observations should be obtained in order to check the method with other examples, particularly, the flare area and thickness.

Acknowledgements. Research at Armagh Observatory is grantaided by the Department of Culture, Arts and Leisure for N. Ireland. This work was supported in part by PPARC grant PPA/G/S/1997/00298. We thank the SAAO for the provision of telescope time and Dr. E. R. Houdebine for useful discussions. We also thank the referee S. L. Hawley for helpful comments.

\section{References}

Bopp, B. W., \& Moffett, T. J. 1973, ApJ, 185, 239

Butler, C. J. 1991, Memorie della Societa Astronomica Italiana, 62, 243

Butler, C. J. 1996, in IAU Colloq. 153, Magnetodynamic Phenomena in the Solar Atmosphere - Prototypes of Stellar Magnetic Activity, 217

Byrne, P. B., \& McKay, D. 1990, A\&A, 227, 490

Cram, L. E., \& Woods, D. T. 1982, ApJ, 257, 269

Dame, L., \& Cram, L. 1983, Sol. Phys., 87, 329

Dame, L., \& Vial, J.-C. 1985, ApJ, 299, L103

Donati-Falchi, A., Falciani, R., \& Smaldone, L. A. 1985, A\&A, 152,165

Doyle, J. G., Butler, C. J., Bryne, P. B., \& van den Oord, G. H. J. 1988, A\&A, 193, 229

Doyle, J. G., Butler, C. J., \& van den Oord, G. H. J. 1989a, A\&A, 208, 208

Doyle, J. G., Byrne, P. B., \& van den Oord, G. H. J. 1989b, A\&A, 224, 153

Doyle, J. G., Jevremović, D., Short, C. I., et al. 2001, A\&A, 369, L13

Doyle, J. G., \& Mathioudakis, M. 1990, A\&A, 227, 130

Doyle, J. G., van der Oord, G. H. J., \& Kellett, B. J. 1992, A\&A, 262, 533

Drake, S. A. 1980, Ph.D. Thesis, 72

Drake, S. A., \& Ulrich, R. K. 1980, ApJS, 42, 351

Foing, B. H., Char, S., Ayres, T., et al. 1994, A\&A, 292, 543

Garcia Alvarez, D. 2000, Irish Astron. J., 27, 117

Gershberg, R. E. 1989, Memorie della Societa Astronomica Italiana, 60, 263
Giampapa, M. S. 1983, in ASSL vol. 102, IAU Colloq. 71, Activity in Red-Dwarf Stars, 223

Gieske, H. A., \& Griem, H. R. 1969, ApJ, 157, 963

Haisch, B., Strong, K. T., \& Rodono, M. 1991, ARA\&A, 29, 275

Hawley, S. L., \& Fisher, G. H. 1992, ApJS, 78, 565

Hawley, S. L., \& Pettersen, B. R. 1991, ApJ, 378, 725

Houdebine, E. R. 1992, Irish Astron. J., 20, 213

Houdebine, E. R., Mathioudakis, M., Doyle, J. G., \& Foing, B. H. 1996, A\&A, 305, 209

Jevremović, D. 1999, Ph.D. Thesis, 187

Jevremović, D., Butler, C. J., Drake, S. A., O’Donoghue, D., \& van Wyk, F. 1998, A\&A, 338, 1057

Johns-Krull, C. M., Hawley, S. L., Basri, G., \& Valenti, J. A. 1997, ApJS, 112, 221

Joy, A. H., \& Wilson, R. E. 1949, ApJ, 109, 231

Katsova, M. M. 1990, Sov. Astron., 34, 614

Katsova, M. M., Livshits, M. A., Butler, C. J., \& Doyle, J. G. 1991, MNRAS, 250, 402

Kundu, M. R., Jackson, P. D., White, S. M., \& Melozzi, M. 1987, ApJ, 312, 822

Kunkel, W. E. 1970, ApJ, 161, 503

Kunkel, W. E. 1973, ApJS, 25, 1

Linsky, J. L., Bornmann, P. L., Carpenter, K. G., et al. 1982, ApJ, 260, 670

Lites, B. W., Neidig, D. F., \& Trujillo Bueno, J. 1986, in The Lower Atmosphere of Solar Flares, Proc. of the Solar Maximum Mission Symp., 101

Mirzoyan, L. V. 1984, Vistas in Astron., 27, 77

Moffett, T. J., \& Bopp, B. W. 1976, ApJS, 31, 61

Montes, D., Saar, S. H., Collier Cameron, A., \& Unruh, Y. C. 1999, MNRAS, 305, 45

Nelson, G. J., Robinson, R. D., Slee, O. B., et al. 1986, MNRAS, 220, 91

Pallavicini, R., Tagliaferri, G., \& Stella, L. 1990, A\&A, 228, 403

Pettersen, B. R. 1989, Sol. Phys., 121, 299

Redman, R. O., \& Suemoto, Z. 1954, MNRAS, 114, 524

Švestka, Z. 1963, Bulletin of the Astronomical Institutes of Czechoslovakia, 14, 234

Švestka, Z. K. 1972, ARA\&A, 10, 1

Tandberg-Hanssen, E., \& Emslie, A. G. 1988, The physics of solar flares (Cambridge and New York, Cambridge University Press), 286

Torczon, V. 1991, SIAM J. Optimization, 1, 123

Torczon, V. 1992, Tech. Report 92-9, Dep. of Mathematical Sciences (Rice University, Houston)

Vidal, C. R. 1964, Zeitschrift Naturforschung Teil A, 19, 947

Wilson, O. C. 1978, ApJ, 226, 379

Worden, S. P. 1983, in ASSL vol. 102, IAU Colloq. 71, Activity in Red-Dwarf Stars, 207

Wuelser, J., \& Marti, H. 1989, ApJ, 341, 1088

Zarro, D. M., \& Canfield, R. C. 1989, ApJ, 338, L33

Zarro, D. M., \& Zirin, H. 1985, A\&A, 148, 240 